\title{
PLANEJAMENTO DO TRANSPORTE NEONATAL: PERCEPÇÃO DAS EOUIPES DO SERVIÇO DE ATENDIMENTO MÓVEL DE URGÊNCIA
}

Aldiania Carlos Balbino¹, Maria Vera Lúcia Moreira Leitão Cardoso², Maria Veraci Oliveira Queiroz ${ }^{1}$

Objetivo: descrever o planejamento do transporte inter-hospitalar de recém-nascido crítico em ambulância sob o olhar dos profissionais do Serviço de Atendimento Móvel de Urgência. Medologia: estudo descritivo, qualitativo, realizado em um Serviço de Atendimento Móvel de Urgência de um município do interior do Ceará, de novembro a dezembro de 2014. Foram entrevistados 17 trabalhadores (médicos, enfermeiros e condutores de veículos) e as informações submetidas à análise temática de conteúdo. Resultados: a categoria Estrutura para execução do transporte neonatal discorre sobre os equipamentos, materiais e veículo e a intitulada Planejamento da segurança na execução do transporte neonatal destaca a comunicação entre as equipes, checagem dos materiais e veículo, o aquecimento da incubadora, o planejamento do deslocamento e a qualificação dos profissionais. Conclusão: para otimizar o planejamento do transporte neonatal em ambulância há necessidade de ampliação de ações e recursos estruturais para se dispensar um transporte seguro e eficaz.

Descritores: Recém-nascido; Ambulâncias; Serviços Médicos de Emergência; Transporte de Pacientes; Pesquisa Qualitativa.

\section{PLANNING OF NEONATAL TRANSPORT: PERCEPTION OF MOBILE EMERGENCY CARE SERVICE TEAMS}

Objective: to describe the planning of inter-hospital transportation of critically ill newborns in ambulance in the perspective of the professionals of the Mobile Emergency Care Service. Methodology: it was a descriptive qualitative study conducted in a Mobile Emergency Care Service of a municipality in the interior of Ceará, Brazil, from November to December 2014. Seventeen workers (physicians, nurses, and drivers) were interviewed and data was submitted to thematic content analysis. Results: the category Structure for performing neonatal transportation addresses the equipment, materials, and vehicle and the one entitled Safety planning in performing neonatal transportation highlights the communication among the teams, verification of materials and vehicle, heating the incubator, planning the displacement, and qualification of professionals. Conclusion: to optimize the planning of neonatal transportation by ambulance, it is necessary to expand the actions and structural resources to ensure a safe and effective transportation.

Descriptors: Infant; Newborn; Ambulances; Emergency Medical Services; Transportation of Patients; Qualitative Research.

\section{PLANIFICACIÓN DEL TRANSPORTE NEONATAL: PERCEPCIÓN DE LOS EQUIPOS DEL SERVICIO DE ATENCIÓN MÓVIL DE URGENCIA}

Objetivo: describir la planificación del transporte inter-hospitalario de recién nacido crítico en ambulancia bajo la mirada de los profesionales del Servicio de Atención Móvil de Urgencia. Metodología: estudio descriptivo, cualitativo, realizado en un Servicio de Atención Móvil de Urgencia de un municipio del interior del Ceará, de noviembre a diciembre de 2014. Fueron entrevistados 17 trabajadores (médicos, enfermeros y conductores de vehículos), cuyas informaciones fueron sometidas al análisis temático de contenidos. Resultados: la categoría Estructura para ejecución del transporte neonatal discurre sobre los equipos, materiales y vehículos; y Planificación de la seguridad en la ejecución del transporte neonatal destaca la comunicación entre los equipos, el chequeo de los materiales y vehículo, calentamiento de la incubadora, planificación del desplazamiento y la cualificación de los profesionales. Conclusión: para optimizar la planificación del transporte neonatal en ambulancia, hay necesidad de ampliación de acciones y recursos estructurales para dispensarse un transporte seguro y eficaz.

Descriptores: Recién Nacido; Ambulancias; Servicios Médicos de Urgencia; Transporte de Pacientes; Investigación Cualitativa. 


\section{INTRODUÇÃO}

O transporte de recém-nascidos (RN) tem sido uma necessidade por muitas décadas, quando se percebeu que nem todos os hospitais poderiam fornecer o nivel de cuidados ao RN prematuro ou gravemente doente ${ }^{(1)}$. Estima-se que em países desenvolvidos 15 a 20\% das crianças nascem em locais sem infraestrutura, em condições precárias de atenção ao parto e nascimento e com carência de serviços especializados. No Brasil, esses percentuais são desconhecidos e podem ser ainda maiores ${ }^{(2)}$.

Apesar dos melhores esforços para providenciar a transferência pré-natal materna quando ditada pelo estado clínico, nem sempre é viável do ponto de vista logístico ou de segurança. Portanto, inevitavelmente, haverá RN que necessitarão de uma transferência urgente ou emergente de um nivel mais baixo de atendimento neonatal para um nivel mais elevado após o nascimento(1).

O transporte neonatal representa uma parte essencial de pacotes de cuidados requeridos pelos serviços perinatais ${ }^{(3)}$. No Brasil, é realizado predominantemente por via terrestre, por entidades públicas e privadas. Por meio da portaria no 2048 do Ministério da Saúde, definiu-se que o Serviço de Atendimento Móvel de Urgência (SAMU) responde pela realização do transporte neonatal e pediátrico cujas condutas deverão obedecer às diretrizes estabelecidas na portaria(4).

O SAMU Cegonha, um dos pontos estratégicos da Rede Cegonha, visa oferecer aos RN críticos que necessitem de transporte inter-hospitalar uma ambulância com incubadora e ventilador de transporte, reforçando a importância da execução do transporte com segurança e qualidade(5). No entanto, apesar dos esforços para a melhoria do transporte neonatal, estudos apontam fragilidades na execução desse serviço de assistência ao RN crítico, destacadamente, falhas na comunicação e a inexistência de infraestrutura mínima para a execução de um transporte seguro(2,6)

Ademais, para garantir um transporte de boa qualidade e seguro, compete aos gestores promoverem melhor integração com os profissionais da assistência para o planejamento e desenvolvimento de ações conjuntas de gestão compartilhada. Considerando a proposta do Ministério da Saúde de trabalhar a segurança do paciente na transferência entre pontos de cuidado(7) e as diretrizes da Rede Cegonha sobre a importância da execução do transporte com segurança e qualidade, a pesquisa parte do questionamento: Qual a percepção das equipes de transporte do SAMU sobre o planejamento do transporte neonatal? O objetivo do estudo foi descrever o planejamento do transporte inter-hospitalar de RN crítico em ambulância sob o olhar dos profissionais do SAMU.

\section{METODOLOGIA}

\section{Tipo de estudo}

Estudo na abordagem qualitativa, descrição das experiências dos participantes.

\section{Participantes da pesquisa}

Participaram 17 trabalhadores (cinco médicos, cinco enfermeiros e sete condutores de ambulância), selecionados de acordo com os seguintes critérios: mínimo de dois anos de experiência no SAMU e ter realizado transporte interhospitalar de RN crítico em ambulância de suporte avançado.

\section{Local do estudo}

Estudo realizado na sede do SAMU de um município do interior do Ceará. Dispõe de uma frota de três ambulâncias, sendo uma Unidade de Suporte Avançado (USA), duas Unidades de Suporte Básico (USB) e uma motolância.

\section{Coleta de dados}

Os dados foram obtidos por meio de entrevista semiestruturada enfocando dados pessoais, tempo de experiência no SAMU e questões relacionadas às ações do planejamento e execução do transporte inter-hospitalar de RN em estado crítico por meio de ambulância.

As entrevistas foram realizadas de novembro a dezembro de 2014 na sede do SAMU, em ambiente privativo, finalizadas quando o pesquisador percebeu que as informações se repetiam, não colaborando com novos elementos sobre a investigação, demonstrando, assim, que as questões da pesquisa estavam contempladas, considerando-se, portanto, a saturação teórica(8).

\section{Procedimentos de análise dos dados}

As entrevistas gravadas em áudio foram transcritas por um dos pesquisadores e submetidas à técnica de análise temática de conteúdo, desenvolvida em três etapas: préanálise; exploração do material; e análise dos dados e interpretação. Na pré-análise, as informações foram lidas exaustivamente e na exploração dos conteúdos estes foram decompostos e codificados em unidades de registro (URs) palavra ou conjunto de palavras que sintetize a ideia de um depoimento, obtendo-se uma visão das características dos resultados $^{(9)}$. Assim, em cada extrato da fala foi destacada sua UR que foram agrupadas em categorias conforme sua afinidade semântica. Por fim, no tratamento dos dados, o pesquisador realizou suas inferências e interpretações.

Os dados pessoais dos trabalhadores foram tabulados em Microsoft Excell versão 2010, sendo verificada média e desvio padrão (DP). 


\section{Procedimentos éticos}

O estudo foi submetido ao Comitê de Ética em Pesquisa da Universidade Federal do Ceará, recebido Parecer $\mathrm{n}$ 은 797.222. Teve anuência dos participantes com assinatura do Termo de Consentimento Livre e Esclarecido e para assegurar o anonimato dos mesmos foram identificados pela inicial da categoria profissional e sequencialmente, o número arábico, conforme aconteciam as entrevistas, exemplo: enfermeiro (El; E2...); médico (M1; M2...); e condutor ( $\mathrm{Cl}$; C2...).

\section{RESULTADOS}

Dos 17 participantes três eram do sexo feminino e 14 do masculino, com idade média de 41,11 anos (DP 7,92). O tempo médio de experiência no SAMU de todos os profissionais investigados foi de 6,47 anos (DP 2,64).

Seguindo o processo analítico descrito, foram apreendidas duas categorias: Estrutura para execução do transporte neonatal e Planejamento da segurança na execução do transporte neonatal.

\section{Estrutura para execução do transporte neonatal}

Os entrevistados destacaram a importância de planejar os equipamentos, materiais e veículo necessários a fim de garantir, durante o transporte, a continuidade da assistência prestada na UTIN. Dentre os materiais citaram aqueles destinados à realização do monitoramento e aquecimento do $\mathrm{RN}$, assistência ventilatória e medicações para o atendimento a possiveis intercorrências.

O material para conseguir empacotar o RN. Para que ele possa ter um conforto similar ao que encontrava no interior do útero materno e tenha um transporte mais agradável [...] Os materiais de apoio à via aérea. Basicamente aqui onde a gente trabalha só tem o hood [...] as drogas de uso para suporte do recém-nato, a bala de oxigênio [...]. (MI)

Temos que ter um oximetro de pulso com bateria resistente para que possamos conduzir essa criança até sair do veículo [...] incubadora de transporte, transparente, de parede dupla, bateria, fonte de luz [...] cilindro de oxigênio[...]balão autoinflável com reservatórios e máscaras, monitor cardio ou oximetro de pulso com bateria, materiais para a intubação traqueal, venóclise e drenagem torácica. Termômetro, estetoscópio, fitas para o controle de glicemia que é um dos fatores que a gente tem que observar na criança [...] as medicações para reanimação caso a criança venha a ter alguma parada. (C5)

Os trabalhadores mencionaram características de uma ambulância de transporte que possibilitasse a assistência ao RN crítico e manutenção do quadro clínico estável.
A gente tem que ver alguns pré-requisitos para o veículo de transporte que seria a altura e o compartimento de paciente suficiente para a acomodação da incubadora com local seguro para a fixação e a presença de tomadas 110 que, muitas vezes, o veículo não está preparado [...] fonte de luz, calor, a fonte de O2, ar comprimido, estoque de gases, espaço interno mínimo para a manipulação do RN [...] ver também alguns ruidos, a vibração. (C5)

\section{[...] Ter as condições da ambulância, aquecimento, amortecedor [...]. (E2)}

Foi mencionada a necessidade de ter uma ambulância exclusiva para o atendimento às transferências interhospitalares do RN crítico, já que, no município investigado, só há uma ambulância de suporte avançado do SAMU para realizar a atividade.

[...] a [ambulância] avançada aqui passa duas horas fazendo um transporte. No mínimo um transporte de um RN a gente mais rápido perde uma hora e meia. Ou seja, uma cidade de 200 mil habitantes ficar duas horas sem uma ambulância avançada é uma coisa que deve ser reavaliada no meu modo de vista[...] poderia ter uma ambulância preparada [exclusiva], com profissionais preparados para fazer esse tipo de transporte e não ocupando uma avançada que seria para toda a cidade. (Cl)

[...] acho que uma ambulância exclusiva para o atendimento de transferências de neonatos graves seria o ideal, pois não ocuparia a única ambulância que temos para atender às demandas de traumas e casos clínicos mais graves. (E4)

\section{Planejamento da segurança na execução do transporte neonatal}

A segurança no transporte foi destacada como ponto importante para o planejamento de um transporte seguro. Destacaram questões relacionadas ao RN, aos materiais e as equipes, enfatizando a necessidade de avaliação do RN antes do transporte e comunicação efetiva entre as equipes e a checagem dos recursos.

A primeira coisa que deve ser feita é avaliar as condições do $R N$. É o ponto número um. Segundo ponto é conversar com o médico que está solicitando o transporte para saber os principais dados do RN e as condições dele para o transporte. Terceiro é ter o contato com o médico que vai receber a criança [...] para saber as condições, para saber se o local já está apto, já está preparado para receber. (M2) 
Outra ação é fazer o checklist do material e do veículo antes da transferência [...]. (E3)

[...] checar o material da ambulância até de preferência antes de sair para verificar se todos os dispositivos que necessitarem ser usados estão dentro da conformidade. Quando houver necessidade de utilizar ventilador mecânico, testar de preferência antes de sair da base. (M3)

Os participantes informaram que os profissionais devem ter habilidade para estabilizar o RN. Além disso, apontaram a participação de profissionais especializados em todas as fases do transporte, desde a solicitação até a condução do RN ao hospital de destino.

Primeiro, profissionais capacitados para estabilizar e transportar o RN com segurança [...]. (E5)

[...] muitas das vezes por ser novato [o profissional], na hora em que um RN venha a dar uma parada cardiorrespiratória ele pelo nervosismo e por ser leigo em termo do transporte[...] ]ele não tomar as decisões corretas que deveriam ser tomadas. Por isso que tem que ter uma equipe muito bem treinada. (Cl)

[...] o ideal seria ambos os médicos, tanto o que solicita a transferência quanto o médico que vai fazer a transferência serem pediatras. Ter um enfermeiro neo e um condutor socorrista com um curso básico para RN [...]. (C4)

Os entrevistados descreveram a relevância de realizar o transporte com a incubadora aquecida de acordo com temperatura programada para o RN. Para isso, afirmaram que o aquecimento deve ser iniciado antes de se alocar o equipamento na ambulância.

Outro ponto importante é a questão da incubadora. A incubadora já estar aquecida antes de entrar na ambulância. É incoerente você colocar uma incubadora que estava desligada sendo que ela vai demorar muito tempo para aquecer. Ela já deve estar previamente aquecida [...]. (M2)

[...] transporte ser feito com incubadora pré-aquecida com temperatura ideal para o RN [...]. (M5)

Segundo os profissionais de saúde, a equipe de transporte deve conhecer e saber manusear os equipamentos utilizados na assistência do RN durante o transporte e que haja treinamento para todos os membros da equipe.

[...] a equipe deve ter treinamento para utilizar os instrumentos e realizar um bom transporte. (C4)

Eu acho que um treinamento da equipe também faz parte do planejamento de todas as transferências. (MI)

Os entrevistados mencionaram também a necessidade de verificar as condições de mobilidade da ambulância para que ocorra o deslocamento da base da ambulância até a unidade de destino do RN.

[...] acho que o principal é planejar como vai ser a viagem [...]. (M4)

[...] os cuidados durante o transporte, em relação ao percurso por onde nós iremos andar. Como é que está o percurso em relação ao trânsito que, muitas vezes, o trânsito tem que colaborar com esse transporte. As condições da via para poder transportar essa criança, a geografia regional, as condições climáticas do tráfego que também é um dos fatores principais. (C5)

\section{DISCUSSÃO}

Evidenciou-se pelo estudo predomínio de participantes do sexo masculino e com relevante experiência no atendimento pré-hospitalar devido ao tempo de serviço relatado, corroborando-se com outros estudos ${ }^{(10-11)}$. A experiência profissional facilita aos profissionais a expertise necessária para o cuidado ao público que aciona o SAMU por queixas clínicas e traumáticas. Em se tratando do transporte neonatal, possibilita o conhecimento e a prática acerca dos quesitos para um manejo mais seguro do RN que necessita de transporte em ambulância.

$\mathrm{Na}$ experiência dos participantes, o planejamento dos materiais é essencial para a garantia da assistência ao RN crítico durante o transporte. Assim, é possivel a continuidade dos cuidados intensivos e atenção a possiveis intercorrências.

Os materiais e equipamentos descritos são utilizados para a manutenção da termorregulação, oxigenoterapia, para o monitoramento do RN além de insumos para o atendimento a intercorrências que necessitem, por exemplo, de drenagem torácica e reanimação. São regulamentados pela Política Nacional de Atenção às Urgências ${ }^{(12)}$ e fazem parte também das diretrizes da Sociedade Brasileira de Pediatria, descritas no Manual de Transporte do RN de Alto Risco, devendo os gestores garanti-los para a execução de uma assistência de qualidade ${ }^{(13)}$

Em relação ao planejamento do veículo, os participantes mencionaram características de uma ambulância de transporte que possibilitasse a assistência ao RN crítico e manutenção do seu quadro clínico estável. Percebe-se 
que o planejamento do veículo envolve processo decisório sobre a capacidade de acomodação de pessoas e espaço para execução de procedimentos, iluminação, refrigeração e suporte para a instalação de equipamentos, havendo necessidade de se estruturar uma coordenação de prétransportes e comunicações ${ }^{(14)}$. Esta poderá dialogar com os gestores sobre as especificações para a aquisição de veículos que atendam as necessidades do público neonatal.

Quanto à disponibilidade de ambulância exclusiva para o transporte de RN críticos, não há determinação nas portarias ministeriais do Brasil. $O$ quantitativo de ambulâncias tipo USA nos SAMUs é baseado em parâmetros populacionais determinados pela portaria 1864/GM, de 29 de setembro de 2003, sendo uma USA a cada 400.000 a 450.000 mil habitantes ${ }^{(12)}$. Outra portaria que define o transporte sanitário de gestantes e de RN de alto risco é a no 1459 intitulada Rede Cegonha, de 24 de junho de 2011, a qual preconiza somente que as USA sejam equipadas com ventiladores neonatais $e$ incubadora(5)

Em relação à comunicação entre as equipes, considera-se uma habilidade imprescindivel aos profissionais de saúde, em destaque os do SAMU, que se comunicam, frequentemente, por telefone móvel ou via rádio, aumentando-se a possibilidade de omissão de informações e possíveis danos à saúde do RN. Em consonância, o Ministério da Saúde afirma que o acesso à informação sobre o estado do paciente na regulação médica é estratégico no planejamento de ações a serem tomadas nas urgências, tornando essas ações mais qualificadas ${ }^{(6)}$.

$\mathrm{Na}$ experiência dos entrevistados é importante para o planejamento do transporte a utilização de checklists pelos profissionais do SAMU, os quais devem ser utilizados no processo de comunicação durante a passagem de plantão e ainda como instrumento de registro para respaldo legal dos profissionais. Salienta-se que estes instrumentos possibilitam ainda a diminuição de erros humanos causados por falha na memória e simplificação do processo de comunicação, visto que é possivel ordenar as informações ${ }^{(15)}$.

Quanto à estabilização do quadro clínico do RN, é primordial que seja garantida antes dele ser acomodado na incubadora de transporte para a prevenção de intercorrências no interior da ambulância. Ressalta-se que o objetivo de todo transporte é transportar o paciente crítico em condições de assepsia, estabilidade térmica, respiratória, metabólica, hidroeletrolítica, hemodinâmica e neurológica, com cuidados executados de forma semelhante aos da unidade de terapia intensiva ${ }^{(16)}$

Entreoscuidadosrelatadospelosprofissionais, evidenciouse a manutenção da incubadora aquecida com valores de temperatura preconizados para o RN. A termorregulação é essenial para o êxito do equilíbrio cardiocirculatório e respiratório logo após o nascimento, sendo importante utilizar estratégias para evitar hipotermia, especialmente em RN prétermo de muito baixo peso(17). Assim, o aquecimento deve ser iniciado antes de se alocar o equipamento na ambulância, sendo este realizado de forma gradual(18), devendo a equipe de transporte conhecer os valores de temperatura aplicados ao RN em uso de incubadora para garantir uma adequada termorregulação.

Os profissionais mencionaram a necessidade de treinamentos para as equipes de transporte que podem ser por meio de simulações, discussão de casos clínicos e uso de tecnologias educacionais, conforme apontado na literatura(1). Um bom conhecimento geral sobre cuidado neonatal e um elevado nível de especialização em habilidades processuais são pré-requisitos para os que efetuam transferências neonatais $^{(3)}$

Apesar de estudos apontarem os benefícios da realização de transportes de RN críticos por especialistas no cuidado neonatal, não há exigência desses profissionais na composição das equipes de transporte do SAMU. Estratégias como o acompanhamento do neonatologista que assiste o RN durante o transporte em ambulância evidenciado em pesquisa executada no SAMU de Recife poderiam melhorar a qualidade do atendimento durante o transporte executado por equipes do SAMU e se alinhar a recomendação da Sociedade Brasileira de Pediatria ${ }^{(19)}$. Mas há de se ponderar que essa é uma proposta de difícil nível de execução devido a carência de pediatras e especialmente neonatologistas nos médios e pequenos centros urbanos.

Para otimizar esse serviço, o médico regulador deve discutir junto a equipe de transporte as condições da malha viária, horário adequado para realização do transporte, solicitar se necessário, apoio da guarda municipal e da polícia para a liberação da via. Deve-se respeitar o limite de $60 \mathrm{~km} /$ hora, minimizando a possibilidade de acidentes automobilísticos, acelerações e desacelerações bruscas e vibrações no interior do veículo que podem acarretar em óbito ou intercorrências clínicas do $\mathrm{RN}^{(13)}$. Nesse sentido, o planejamento do deslocamento em ambulância foi apontado pelos profissionais como medida para garantir a segurança do transporte do RN.

\section{Limitações do estudo}

O estudo apresenta limitação por ter sido realizado em uma unidade do SAMU localizada em uma cidade de médio porte, com somente uma USA para realizar as transferências neonatais. Portanto, a realidade não foi apreendida em outros contextos. 


\section{Contribuições para a prática}

Apresentam-se pontos estratégicos acerca do gerenciamento do cuidado do RN que necessita de transporte inter-hospitalar por meio de ambulância, reduzindo-se a lacuna de conhecimento em relação à temática e um maior suporte para a melhoria das práticas de saúde. Apontamse também competências requeridas pelos profissionais envolvidos, em destaque os enfermeiros, as quais precisam ser fortalecidas durante o processo de formação e nas sessões de educação em serviço.

\section{CONSIDERAÇÕES FINAIS}

A pesquisa mostra a diversidade de fatores implicados na realização do transporte de $\mathrm{RN}$ críticos, e fundamentalmente, o planejamento do veículo, a provisão de equipamentos e materiais a serem utilizados pela equipe de transporte treinada, além da utilização de uma efetiva comunicação com todos os envolvidos na ação.
Recomenda-se o desenvolvimento de pesquisas em grandes centros urbanos para averiguar se o planejamento do transporte engloba outros recursos, explorando como o componente Transporte Sanitário e Regulação da Rede Cegonha está sendo planejado e executado nos estados brasileiros. Considera-se que para otimizar o planejamento do transporte neonatal em ambulância há necessidade de ampliação de ações e recursos estruturais para se dispensar um transporte seguro e eficaz.

\section{Contribuição dos autores:}

Aldiania Carlos Balbino: Concepção e desenho, análise e interpretação dos dados, redação do artigo, revisão crítica, revisão final. Maria Vera Lúcia Moreira Leitão Cardoso: Concepção e desenho, análise e interpretação dos dados, revisão crítica e revisão final. Maria Veraci Oliveira Queiroz: Análise e interpretação dos dados, redação do artigo, revisão crítica e revisão final. 


\section{REFERÊNCIAS}

1. Diehl BC. Neonatal transport: current trends and practices. Crit Care Nurs Clin North Am 30 [Internet]. 2018 [cited 2019 May 23]:30(4):597-606. Available from: https://linkinghub.elsevier.com/retrieve/pii/S0899-5885(18)30982-1

2. Pimenta PCO, Alves VH. Interhospital transport of the high-risk newborn: a challenge for the nursing staf. Cogitare Enferm [Internet]. 2016 [cited 2019 May 23];21(n.esp):1-9. Available from: http://revistas. ufpr.br/cogitare/article/view/45047/pdf_1

3. McEvoy CG, Descloux E, Barazzoni MS, Diaw CS, Tolsa JF, Roth-Kleiner M. Evaluation of neonatal transport in Western Switzerland: a model of perinatal regionalization. Clin Med Insights Pediatr [Internet]. 2017 [cited 2019 May 23];11:1179556517709021. Available from: https:// www.ncbi.nlm.nih.gov/pmc/articles/PMC5439992/

4. Ministério da Saúde (BR). Secretaria de Atenção à Saúde, Coordenação Geral de Urgências e Emergências. Portaria GM/MS no 2048, de 5 de novembro de 2002. Brasilia: Ministério da Saúde; [Internet]. 2002 [cited 2019 May 23]. Available from: http://bvsms.saude.gov.br/ bvs/saudelegis/gm/2002/prt2048_05_11_2002.html

5. Oliveira FAM, Leal GC, Wolff LDG, Rabelo M, Poliquesi CB. Reflections on the nurse's role in the rede cegonha (stork Network). Rev Enferm UFPE on line [Internet]. 2016 [cited 2019 May 23];10(Supl.2):867-74. Available from: https://periodicos.ufpe.br/revistas/revistaenfermagem/article/view/11030/12420

6. Balbino AC, Cardoso MVLML. Difficulties in critical newborn interhospital transport carried out by the Emergency Mobile Care Services. Texto Contexto Enferm [Internet]. 2017 [cited 2019 May 23]:26(3):e0790016. Available from: http://www.scielo.br/pdf/tce/ v26n3/en_0104-0707-tce-26-03-e0790016.pdf

7. Ministério da Saúde (BR). Portaria $n^{\circ} 529$, de 1 ㅇ de abril de 2013: institui o Programa Nacional de Segurança do Paciente (PNSP). Brasilia: Ministério da Saúde [Internet]. 2013 [cited 2019 May 23]. Available from: http://bvsms.saude.gov.br/bvs/saudelegis/gm/2013/ prt0529_01_04_2013.html

8. Barcellos FBJ, Moretti LB, Borges SMG, Janete R, Ribeiro TE, Gusmão MD. Amostragem em pesquisas qualitativas: proposta de procedimentos para constatar saturação teórica. Cad Saúde Pública [Internet]. 2011 [cited 2019 May 23]:27(2):389-94. Available from: http://www. scielo.br/pdf/csp/v27n2/20.pdf

9. Bardin L. Análise de conteúdo. Porto (PT): Edições 70; 2011.

10. Silva FG, Andrade AP, Ponte KMA, Ferreira VES, Sousa BS, Gonçalves KG. Predisposição para Sindrome de Burnout na equipe de enfermagem do Serviço de Atendimento Móvel de Urgência. Enferm Foco [Internet]. 2019 [cited 2019 May 23];10(1):40-45. Available from: http:// revista.cofen.gov.br/index.php/enfermagem/article/view/1600/491
11. Luz LM, Torres RRB, Sarmento KMVQ, Sales JMR, Farias KN, Marques MB. Sindrome de burnout em profissionais do serviço de atendimento móvel de urgência. Rev Pesqui Cuid Fundam Online [Internet]. 2017 [cited 2019 May 23]:9(1):238-46. Available from: http:// seer.unirio.br/index.php/cuidadofundamental/article/view/5400

12. Ministério da Saúde (BR). Portaria GM/MS n이일 de 20 de setembro de 2003. Institui o componente pré-hospitalar móvel da Política Nacional de Atenção às Urgências, por intermédio da implantação do serviço de Atendimento Móvel de Urgência em municipios e regiões de todo o território brasileiro: SAMU-192. Brasilia: Ministério da Saúde [Internet] 2010 [cited 2019 May 23]. Available from: http://bvsms.saude.gov.br/bvs/saudelegis/gm/2003/prt1864_29_09_2003.html

13. Marba ST, Caldas JPS, Nader PJH, Ramos JRM, Machado MGP Almeida MFB, et al. Transporte do recém-nascido de alto risco: diretrizes da Sociedade Brasileira de Pediatria. Rio de Janeiro (RJ): Sociedade Brasileira de Pediatria; 2017.

14. Roy MP, Gupta R, Sehgal R. Neonatal transport in India: From public health perspective. Med J DY Patil Univ [Internet]. 2016 [cited 2019 May 23]:9(Issue 5):566-9. Available from: http://www.mjdrdypu.org/ temp/MedJDYPatilUniv95566-2876015_075920.pdf

15. Oliveira MCB, Korb A, Zocche DAA, Bezerra DC, Pertille F, Frigo J. Adesão do cheklist cirúrgico à luz da cultura de segurança do paciente. Rev SOBECC [Internet]. 2018 [cited 2019 May 23];23(1):36-42. Available from: http://docs.bvsalud.org/biblioref/2018/04/882693/ sobecc-v23n1_pt_36-42.pdf

16. Henry S, Trotman H. Challenges in neonatal transport in Jamaica: A resource-limited setting. J Trop Pediatr [Internet]. 2017 [cited 2019 May 23]:63(4):307-13, Available from: https://academic.oup.com/tropej/article-lookup/doi/10.1093/tropej/fmw095

17. Caldas JPS, Millen FC, Camargo JF, Castro PAC, Camilo ALF, Marba STM. Effectiveness of a measure program to prevent admission hypothermia in very low-birth weight preterm infants. J Pediatr (Rio J) [Internet]. 2018 [cited 2019 May 23]:94(4):368-73. Available from: http:// www.scielo.br/pdf/jped/v94n4/0021-7557-jped-94-04-0368.pdf

18. Costa CC, Tonete VLP. Parada CMGL. Knowledge and practices regarding the handling of neonatal incubators among nursing professionals. Acta Paul Enferm [Internet]. 2017 [cited 2019 May 23];30(2):174-80. Available from: http://www.scielo.br/pdf/ape/v30n2/ en_1982-0194-ape-30-02-0174.pdf

19. Romanzeira JCF, Sarinho SW. Quality assessment of neonatal transport performed by the Mobile Emergency Medical Services (SAMU). J Pediatr [Internet]. 2015 [cited 2019 May 23]:91(4):380-5. Available from: http://www.scielo.br/pdf/jped/v9ln4/0021-7557jped-91-04-00380.pdf 